\title{
PRESENCIA DE PTYCHODUS MORTONI (ELASMOBRANCHII: PTYCHODONTIDAE) EN EL CRETÁCICO SUPERIOR DE VENEZUELA
}

\author{
PRESENCE OF PTYCHODUS MORTINI \\ (ELASMOBRANCHII: PTYCHODONTIDAE) IN THE UPPER CRETACEOUS \\ OF VENEZUELA
}

\author{
Jorge D. Carrillo-Briceño \\ Programa de Magíster en Oceanografía, Universidad de Valparaíso. Avenida \\ Borgoño 16344, Reñaca, Viña del Mar, Chile \\ Jorgedcb100@gmail.com
}

(Recibido: 9/04/2012 ; aceptado: 11/06/2012)

\begin{abstract}
Ptychodus mortoni is a durophagous shark characteristic from the Upper Cretaceous, with a chronostratigraphic record between the Turonian-Maastrichtian. The present contribution illustrates and describes a set of teeth of Ptychodus mortoni from the La Luna Formation, Upper Cretaceous of the Sierra de Perijá, Zulia State, Venezuela. This material represents the only known record for South America, increasing the paleobiogeographi distribution of the taxon, which was previously known only from Africa, North America and Europe.

Key word: Chondrichthyes, Late Cretaceous, La Luna Formation, Sierra de Perijá, Venezuela.

RESUMEN: Ptychodus mortoni es un tiburón durofago característico del Cretácico Superior, con un registro cronoestratigráfico entre el Turoniano- Maastrichtiano. En la presente contribución se ilustra y describe un conjunto de dientes de un mismo ejemplar de Ptychodus mortoni, proveniente de la Formación La Luna, del Cretácico Superior de La Sierra de Perijá, Estado Zulia, Venezuela. Este material representa el único registro conocido de esta especie para América del sur y extiende la distribución paleobiogeográfica del taxón, el cual era conocido sólo para África, América del Norte y Europa.

Palabras clave: Chondrichthyes, Cretácico Superior, Formación La Luna, Sierra de Perijá, Venezuela.
\end{abstract}


Ptychodus Agassiz 1835, es un género de tiburones característicos del Cretácico Superior, con una distribución cronoestratigráfica que va desde el Albiense hasta el Maestrichtiense (Johnson et al., 2002). El género Ptychodus presenta una morfología dental adaptada para la trituración, lo que ha llevado a pensar que los representantes de este género poseían hábitos alimenticios basados en moluscos (Cappetta, 1987; Shimada et al., 2010). En la actualidad se conocen poco más de 12 especies con registros en África, America del Norte y del Sur, Asia, Australia, Europa y El Caribe (Reinhart, 1951; Wenz, 1972; Cappetta, 1987; Williamson et al., 1989; Welton \& Farish, 1993; Antunes \& Cappetta, 2002; Brito \& Janvier, 2002; Mutter et al., 2005). La posición sistemática de Ptychodus todavía es muy controvertida. Se ha propuesto que estos tiburones deben ser incluidos tanto en el grupo de los hybodontiformes (Cappetta, 1987; Welton \& Farish, 1993), como en el de los Neoselachii (Maisey et al., 2004).

Ptychodus mortoni Mantell 1839, es una de las especies mejor conocidas y su registro incluye el Cretácico Superior de África, América del Norte y Europa, con una distribución cronoestratigráfica que va desde el Turoniano Temprano hasta el Maastrichtiano (Cappetta, 1987; Alvarado-Ortega et al., 2006; Blanco-Piñón et al., 2007; Shimada et al., 2010). La mayoría de los registros de P. mortoni se limitan a dientes aislados; sin embargo, también se conocen algunos registros excepcionales, representados por placas dentales completas del Cretácico Superior de Kansas, en América del Norte. Algunos de estos ejemplares fósiles han permitido hipotetizar tamaños de hasta $10 \mathrm{~m}$ para este tiburón (Shimada et al., 2010). El único registro conocido de P. mortoni para América del Sur fue reportado por Aguilera \& Lundberg (2010) y Aguilera (2010), para el Cretácico Superior de Venezuela. El material de Venezuela proviene de la Sierra de Perijá, en el Estado Zulia (Fig. 1), pero la ubicación exacta del hallazgo se desconoce. Según Aguilera (2010), el material referido a P. mortoni corresponde a la Formación La Luna.
La presente contribución tiene por objeto describir e ilustrar el material de P. mortoni mencionado por Aguilera \& Lundberg (2010) y Aguilera (2010), así como discutir las implicancias paleobiogeográficas que este registro representa.

\section{SISTEMÁTICA}

\author{
Clase Chondrichthyes Huxley, 1880 \\ Orden incertae sedis \\ Familia Ptychodontidae Jaekel, 1898 \\ Genero Ptychodus Agassiz, 1835 \\ Ptychodus mortoni, Mantell 1839
}

Fig. 2 A-D

Material referido: Cinco dientes completos sueltos; dos dientes completos y veinte dientes fragmentados contenidos en una concreción calcárea; todo el material está depositado en la Colección de Paleontología de la Alcaldía de Urumaco en el Estado Falcón bajo el código CURS-211.

Edad y Geología: Los ejemplares aquí tratados provienen de los afloramientos de la Formación La Luna en la Sierra de Perijá, estado Zulia. Debido al amplio rango cronoestratigráfico (Cenomaniano - Campaniano) de esta unidad geológica en el occidente de Venezuela (Zapata et al., 2003) y dado que se desconoce la localidad exacta del hallazgo, no es posible precisar con mayor detalle la edad de los ejemplares. En la Sierra de Perijá la Formación La Luna esta suprayaciendo al Grupo Cogollo, el cual tiene una edad Aptiense-Albiense; sin embargo, autores como Erlich et al., (1998), plantean que para la zona existe un hiatus regional de casi todo el Cenomaniano para la base de la Formación La Luna, quedando esta unidad geológica asignada en la región con un rango cronoestratigráfico entre el Cenomaniano Superior y el Santoniano, donde existe un cambio transicional hacia la suprayacente Formación Socuy (Campaniano). 


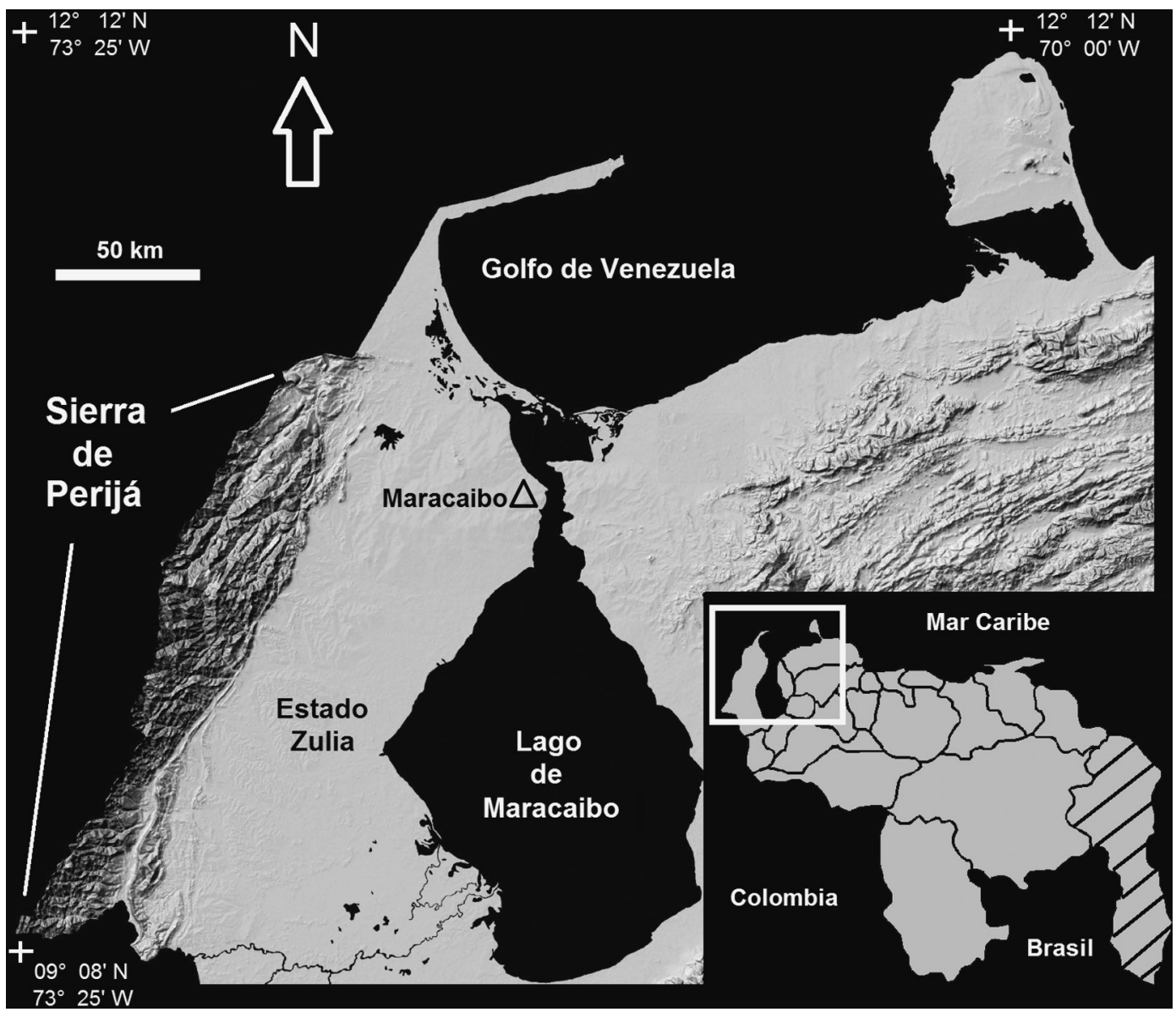

Fig. 1: Situación relativa de la Sierra de Perijá en el Occidente de Venezuela, Estado Zulia (modificado de Garrity et al., 2004).

Descripción: se describen aquí los cinco dientes completos que se hayan desprendidos de la matriz. Estos son considerados como representativos del conjunto y su condición de preservación permite describirlos en forma detallada.

Se trata de dos dientes postero laterales y tres antero mediales. Todos poseen la corona con una cúspide en forma de domo. De dicha cúspide parten una serie de fuertes crestas que se distribuyen radialmente hasta el área marginal, donde se hacen más finas y forman un patrón reticular. El ejemplar más grande tiene $25 \mathrm{~mm}$ de ancho, 19 $\mathrm{mm}$ de largo y una altura de la corona de $14 \mathrm{~mm}$.

\section{DISCUSIÓN}

Según Cappetta (1987), los dientes de Ptychodus mortoni poseen una morfología coronaria caracterizada por una cúspide cónica de donde parte en forma radial una serie de fuertes crestas que en el área marginal se hacen más finas y adquieren un patrón reticular. Estas características de la corona son diagnósticas de esta especie (Cappetta, 1987).

Dentro del género Ptychodus, los ejemplares más similares a $P$. mortoni corresponden a los taxones $P$. cyclodontis, del Turoniano de Cuba 


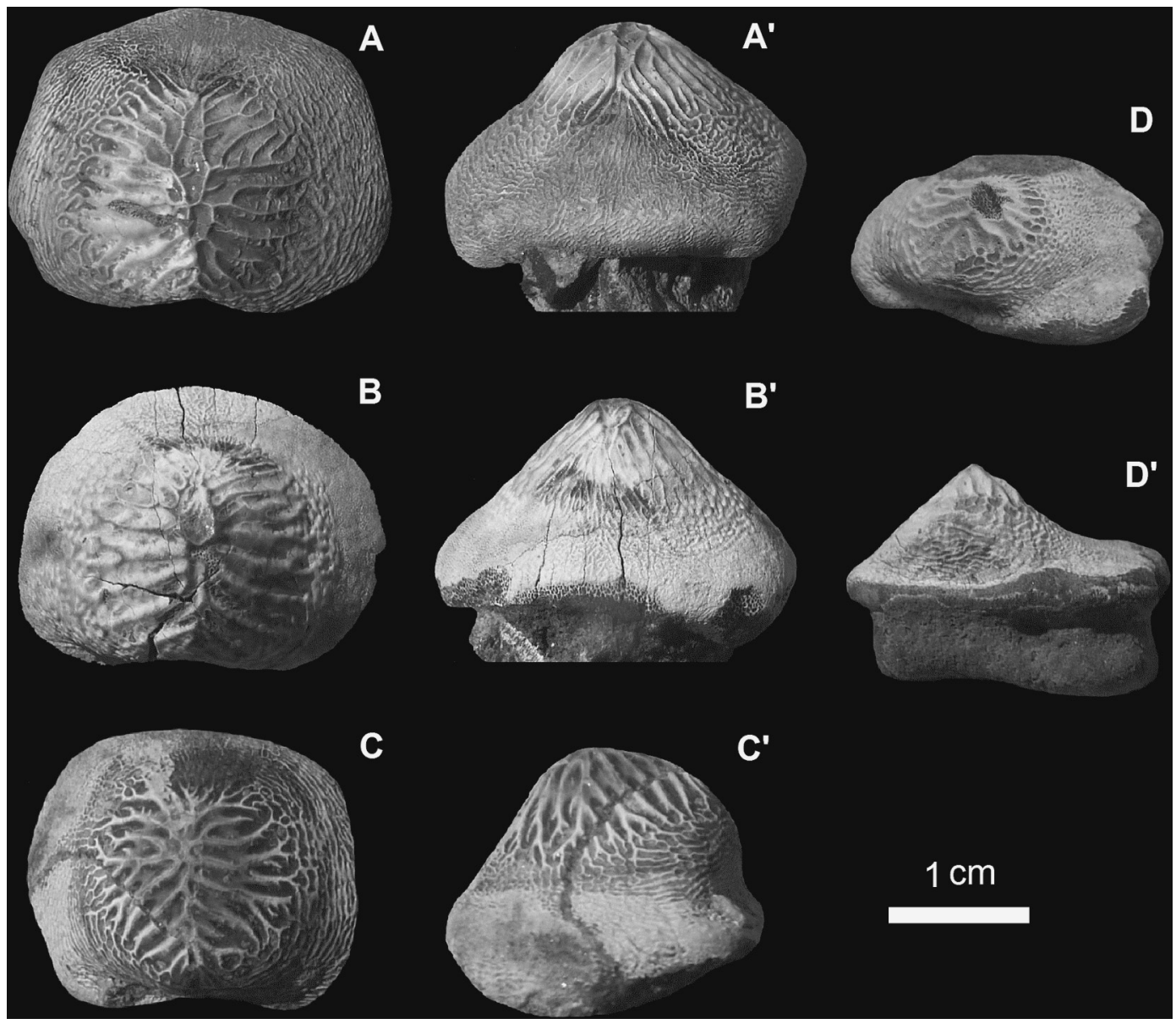

Fig. 2: A-D, Ptychodus mortoni (CURS-211). A-C, dientes anterior medial en vista oclusal, en vista labial (A', B') y en vista lingual (C'). D, diente posterior lateral en vista oclusal y en vista lingual (D').

(Mutter et al., 2005); Ptychodus sp., presumiblemente de la Formación La Luna, en Venezuela (Brito \& Janvier, 2002) y Ptychodus sp., procedente de la Formación La Luna en el estado Trujillo, en los Andes de Venezuela (Carrillo, 2009). Sin embargo, los ejemplares mencionados de Ptychodus cyclodontis y Ptychodus sp. se diferencian de CURS-211 por poseer una dentición homodonta (Brito \& Janvier, 2002; Mutter et al., $2005)$ vs. dentición heterodonta en P. mortoni.

Los ejemplares de Ptychodus sp. descritos para la Formación La Luna (Carrillo, 2009) presentan una variabilidad en tamaños y formas que llevarían a pensar que pertenecen a un conjunto dentario heterodonto muy similar a los dientes cónicos antero laterales de P. mortoni (Welton \&
Farish, 1993). Sin embargo, los dientes de P. mortoni presentan una corona más redondeada y no tan aguda como los descritos por Carrillo (2009). Los caracteres morfológicos coronarios observados en los dientes CURS-211 y su patrón heterodonto permiten ratificar la asignación de estos ejemplares a P. mortoni.

La distribución paleobiogeográfica y cronoestratigráfica de Ptychodus mortoni conocida hasta el momento incluye África, en el Turoniano Superior de Angola (Antunes \& Cappetta, 2002); América del Norte, en el Coniaciano-Campaniano de Estados Unidos de Norte América (Cappetta, 1987; Williamson et al., 1989; Shimada et al., 2010) y el Turoniano Temprano-Maastrichtiano de México (Alvarado-Ortega et al., 2006; Blanco- 
Piñón et al., 2007) y Europa, en el ConiacianoCampaniano de Bélgica, Inglaterra e Italia (Blanco-Piñón et al., 2007).

\section{CONCLUSIONES}

Aguilera \& Lundberg (2010) y Aguilera (2010) asignaron provisoriamente los ejemplares CURS211 a la especie $P$. mortoni. En base a la descripción y a las comparaciones aquí establecidas es posible confirmar dicha asignación. Adicionalmente, se propone una edad para estos ejemplares entre el Cenomaniano Superior y el Santoniano, con base en el rango cronoestratigráfico de la Formación La Luna en la Sierra de Perijá. La presencia de Ptychodus mortoni en el Cretácico superior de Venezuela, representa el único registro conocido de la especie para el Mesozoico de América del Sur, ampliando notablemente la distribución geográfica de este taxón.

\section{AGRADECIMIENTOS}

El autor quiere agradecer al Dr. Orangel Aguilera (Museo Paraense Emílio Goeldi, Belem, Brasil) y al Lic. Sergio Bogan (Fundación de Historia Natural "Félix de Azara", Universidad Maimónides, Buenos Aires, Argentina) por sus valiosos aportes en el desarrollo de esta investigación.

\section{REFERENCIAS}

AGUilerA, O., 2010: Peces Fósiles del Caribe de Venezuela.- 258 págs. Gorham Printing, Centralia, USA.

AGUILERA, O. \& LUNDBERG, J., 2009: Venezuelan caribbean and orinocoan neogene fish.- En: SÁNCHEZ-VILLAGRA, M., AGUILERA, O. \& CARLINI, F. (eds): Urumaco and Venezuelan paleontology- The fossil record of the northern neotropics.- Indiana Press University: 129-152.
ANTUNES, M.T. \& CAPPETTA, H., 2002: Sélaciens du Crétacé (Albién-Maastrichtien) d'Angola.Palaeontographica, Abtheilung A, 264: 85-146.

ALVARADO-ORTEGA, J., GARIBAYROMERO, L.M., BLANCO-PIÑÓN, A., GONZÁLEZ-BARBA, G., VEGAVERA, F. J. \& CENTENO-GARCÍA, E., 2006: Los peces fósiles de la Formación Mexcala (Cretácico Superior) en el Estado de Guerrero, México.- Rev. Brasileira de Paleont. 9(3): 261-272.

BLANCO-PIÑÓN, A., GARIBAY-ROMERO, L.M. \& ALVARADO-ORTEGA, J., 2007: The oldest stratigraphic record of the Late Cretaceous shark Ptychodus mortoni Agassiz, from Vallecillo, Nuevo León, northeastern Mexico.- Rev. Mexicana de Cienc. Geol. 24(1): 25-30.

BRITO, P.M. \& JANVIER, P., 2002: A ptychodontid (Chondrichthyes, Elasmobranchii) from the Upper Cretaceous of South America.Geodiversitas, 24(4): 785-790.

CAPPETTA, H., 1987: Chondrychthyes II. Mesozoic and Cenozoic Elasmobranchii.En: Schultze, H. P (ed.): Handbook of Paleoichthyology, Vol. 3B. Gustav Fisher Verlag, Stuttgart, 193 págs.

CARRILlO, J., 2009: Presencia del genero Ptychodus (Elasmobranchii: Ptychodontiade) en el Cretácico Superior de los Andes de Trujillo Venezuela.Geominas, 37(50): 207-210.

ERLICH, R.N., MACSOTAY, O., NEDERBRAGT A.J. \& LORENTE, M.A., 1999: Palaeoceanography, palaeoecology, and depositional environments of Upper Cretaceous rocks of western Venezuela.- Palaeogeography, Palaeoclimatology, Palaeoecology, 153: 203- 238. 
GARRITY, C.P, HACKLEY, P.C. \& URBANI, P.F., 2004: Digital Shaded-relief map of Venezuela (Version 1.0).[Reston, Va.].U.S. Geological Survey.

JOHNSON, S.C., LUCAS, S.G. \& FRIEDMAN, V., 2002: Stratigraphic range and bioestratigraphic utility of the Cretaceous shark genus Ptychodus.- Geol. Soc. Amer., Annual meeting: Denver, Colorado, USA, Abstracts Programs, 34(6): 462.

MAISEY J.G., NAYLOR G.J.P. \& WARD D.J., 2004: Mesozoic elasmobranchs, neoselachian phylogeny and the rise of modern elasmobranch diversity.- En: ARRATIA, G. \& TINTORI, A. (eds.): Mesozoic fishes 3- systematics, paleoenvironment and biodiversity.- Verlag Dr. Friedrich Pfeil, München: 17-56.

MUTTER , R.J., ITURRALDE-VINENT, M. \& CARMONA, J.F., 2005: The first Mesozoic Caribbean shark is from the Turonian of Cuba: Ptychodus cyclodontis sp. nov. (? Neoselachii).- J. Verteb. Paleont. 25(4): 976-978.

REINHART, R.T., 1951: A new shark of the family Ptychodontidae from South America.University of California Publications, Bull. Geol. Sci. 28: 195-202.
SHIMADA, K., EVERHART, M.J., DECKER, R. \& DECKER, P., 2010: A new skeletal remain of the durophagous shark, Ptychodus mortoni, from the Upper Cretaceous of North America: an indication of gigantic body size.- Cretaceous Res. 31: 249-254.

WENZ, S., 1972: Présence du Sélacien Ptychodus (Pt. chappelli) dans le Crétacé supérieur de l'Équateur (Amérique du Sud).- Bull.Mus. Nat. d'Hist. Naturelle, 74: 91-94.

WELTON, B. J. \& FARISH, R.F., 1993: The Collector's Guide to Fossils Sharks and Rays from the Cretaceous of Texas.- 204 págs. Before Time, Lewisvile, Texas.

WILLIAMSON, T.E., LUCAS, S.G. \& PENCE, R.,1989: Selachians from the Hosta Tongue of the Point Lookout Sandstone (Upper Cretaceous, Santonian), Central New Mexico.New Mexico Geol. Soc. 40: 239-245.

ZAPATA, E., PADRO, V., MADRID, I., KERTZNUS, V., TRUSKOWSKI, I. \& LORENTE, M.A., 2003: Biostratigraphic, sedimentologic, and chemostratigraphic study of the La Luna Formation (Late Turonian-Campanian) in the San Miguel and Las Hernández sections, western Venezuela.- Palaios, 18(4): 367-377. 